\title{
Ultrathin metasurface with high absorptance for waterborne sound
}

Jun Mei, Xiujuan Zhang, and Ying Wu

Citation: Journal of Applied Physics 123, 091710 (2018);

View online: https://doi.org/10.1063/1.5009382

View Table of Contents: http://aip.scitation.org/toc/jap/123/9

Published by the American Institute of Physics

\section{Scilight}

Sharp, quick summaries illuminating the latest physics research 


\title{
Ultrathin metasurface with high absorptance for waterborne sound
}

\author{
Jun Mei, ${ }^{1}$ Xiujuan Zhang, ${ }^{2}$ and Ying $\mathrm{Wu}^{2, a)}$ \\ ${ }^{1}$ Department of Physics, South China University of Technology, Guangzhou 510640, China \\ ${ }^{2}$ King Abdullah University of Science and Technology (KAUST), Division of Computer, Electrical and \\ Mathematical Sciences and Engineering, Thuwal 23955-6900, Saudi Arabia
}

(Received 16 October 2017; accepted 20 December 2017; published online 12 January 2018)

\begin{abstract}
We present a design for an acoustic metasurface which can efficiently absorb low-frequency sound energy in water. The metasurface has a simple structure and consists of only two common materials: i.e., water and silicone rubber. The optimized material and geometrical parameters of the designed metasurface are determined by an analytic formula in conjunction with an iterative process based on the retrieval method. Although the metasurface is as thin as 0.15 of the wavelength, it can absorb $99.7 \%$ of the normally incident sound wave energy. Furthermore, the metasurface maintains a substantially high absorptance over a relatively broad bandwidth, and also works well for oblique incidence with an incident angle of up to $50^{\circ}$. Potential applications in the field of underwater sound isolation are expected. Published by AIP Publishing.

https://doi.org/10.1063/1.5009382
\end{abstract}

\section{INTRODUCTION}

The efficient absorption of low-frequency acoustic waves propagating in water has been both an interesting scientific problem and a challenging engineering task for a long time. Most natural solid materials (such as metals and rocks) are dense and hard, with mass densities usually several times that of water, causing a high impedance contrast between these solid materials and water. Therefore, efficiently absorbing sound waves in water using homogeneous solid materials are generally considered a difficult task, because a significant portion of the incident wave energy would be reflected back when it reached the interface between water and an absorber made from solid materials.

Recently, an electromagnetic metasurface - a thin layer of artificial material of subwavelength thickness-has attracted much attention because it can provide unusual electromagnetic properties on demand. ${ }^{1-3}$ In a way, a metasurface can be viewed as a two-dimensional version of a metamaterial, because both exhibit unusual properties. Soon, the idea of a metasurface was adopted for acoustic waves, and different kinds of acoustic metasurfaces, either structured or non-structured with subwavelength-scaled patterns, were designed. ${ }^{4-26}$ It was both theoretically and experimentally demonstrated that acoustic metasurfaces can be used to control the wavefront of reflected and/or transmitted waves by imparting a gradient phase shift to the incoming waves, and many interesting applications such as planar lenses and extraordinary reflection have been demonstrated. ${ }^{4-29}$ One important category of acoustic metasurfaces is the absorptive metasurface, which exhibits high absorptances for incident acoustic waves and could lead to promising applications in the fields of noise cancellation and isolation. ${ }^{30-46}$

Although most absorptive acoustic metasurfaces are designed for airborne sound, ${ }^{30-45}$ very few acoustic metasurfaces are aimed at efficiently absorbing low-frequency sound

\footnotetext{
${ }^{\text {a) }}$ Author to whom correspondence should be addressed: ying.wu@kaust.edu.sa
}

in water. In one acoustic metasurface, air bubbles are intentionally introduced/incorporated into the solid components of an inhomogeneous structure to mediate the impedance mismatch. ${ }^{46}$ As a result of the matched impedance, ${ }^{25,46}$ very little wave energy is reflected at the interface between water and the metasurface, and high absorptance is achieved.

In this work, we propose a new design for a metasurface to absorb low-frequency acoustic wave energy in water. The metasurface has a simple structure and comprises only common materials, such as water and silicone rubber. We derive an analytic formula for absorption in a two-layer non-transparent system. As reflectance approaches zero, nearly total absorption is achieved. Applying this analytic formula, we obtain a combination of the material parameters and the thickness of each layer, giving rise to maximum absorption in the low-frequency regime. However, the desired material parameters of one layer do not fit any known natural materials. Therefore, we develop an effective medium theory that can guide us in designing a suitable artificial structure using ordinary materials to achieve the desired effective parameters. We further use an iterative method to optimize the absorption performance of the structure and obtain an ultrathin metasurface, whose thickness is around 0.15 of the wavelength, to efficiently absorb low-frequency sound in water over a relatively broad bandwidth. The metasurface can nearly totally absorb the incident wave energy for oblique incidence with an incident angle of up to $50^{\circ}$.

\section{THEORETICAL ANALYSIS}

The metasurface immersed in water is composed of two thin layers of absorbing materials placed in front of a reflective surface, as shown schematically in Fig. 1(a). The sound velocity, mass density and thickness of the layers are denoted as $c_{i}, \rho_{i}$, and $h_{i}$, respectively. The subscript $i=1,2$ represents the layer index. The mass density and the sound velocity for water are $\rho_{0}=1000 \mathrm{~kg} / \mathrm{m}^{3}$ and $c_{0}=1490 \mathrm{~m} / \mathrm{s}$, respectively. Here, we choose two layers to construct the metasurface for 


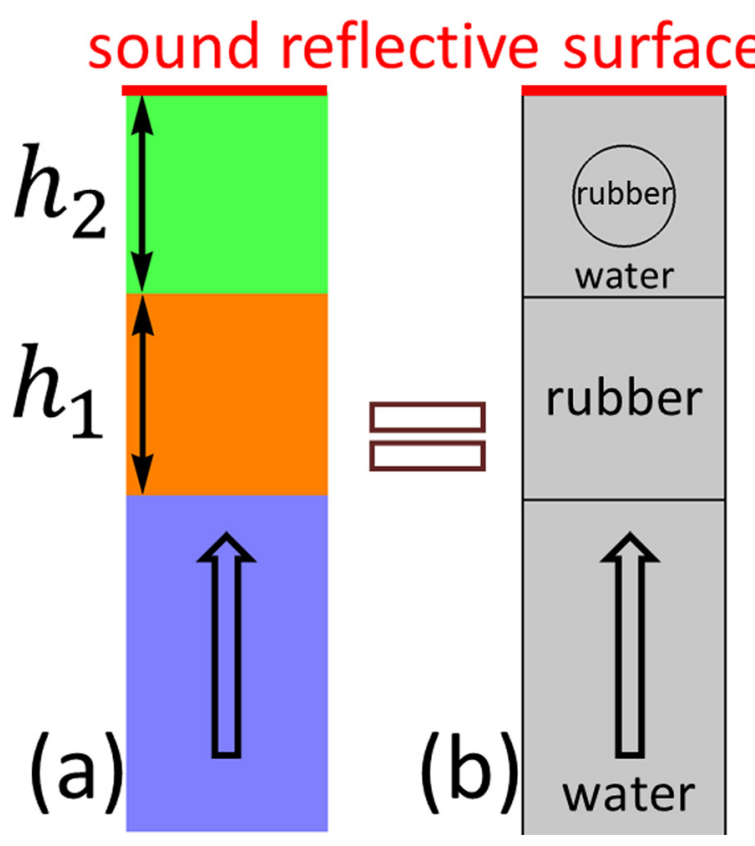

FIG. 1. (a) A schematic view of the absorptive acoustic metasurface, which consists of a thin film with thickness $h_{1}$ (in orange) and a thin medium with thickness $h_{2}$ (in green) placed in front of a reflective surface (in red). The sound wave is incident from the water domain (in purple), as represented by an arrow. (b) A realistic structure to realize the proposed metasurface, where the homogeneous film is silicone rubber, and the heterogeneous medium is composed of a periodic row of rubber cylinders (with radius $r_{2}$ ) placed in a water background.

the following reasons: first, a two-layer metasurface offers more degrees of freedom than a one-layer one to adjust the impedance, to avoid a large portion of the incident wave being reflected; second, the two-layer structure is simpler and may also be thinner than multiple-layer structures.

For a plane wave normally incident onto the metasurface, as depicted in Fig. 1(a), the amplitude reflection coefficient $R$ can be evaluated as

$$
R=\frac{Z_{\text {in }}-Z_{0}}{Z_{\text {in }}+Z_{0}}
$$

where $Z_{0}=\rho_{0} c_{0}$ is the specific acoustic impedance of water and $Z_{\text {in }}$ is the input impedance of the metasurface: i.e., the impedance felt by the incident wave at the interface between water and the metasurface. After applying appropriate boundary conditions on the interfaces between adjacent layers-i.e., the continuity of the pressure and the normal velocity across the interfaces-we obtain

$$
Z_{\text {in }}=\frac{Z_{2}-Z_{1} \tan \phi_{1} \tan \phi_{2}}{-i\left(Z_{1} \tan \phi_{2}+Z_{2} \tan \phi_{1}\right)} Z_{1},
$$

where $Z_{1}$ and $Z_{2}$ are the specific acoustic impedances of layers 1 and 2 , respectively; $\phi_{1}=k_{1} h_{1}$ and $\phi_{2}=k_{2} h_{2}$ are the accumulated phase changes of the acoustic wave when it travels through layers 1 and 2, with $k_{1}$ and $k_{2}$ being the corresponding wavenumbers. Inserting Eq. (2) into Eq. (1), we obtain

$$
R=\frac{Z_{1}\left(Z_{2}-Z_{1} \tan \phi_{1} \tan \phi_{2}\right)+i Z_{0}\left(Z_{1} \tan \phi_{2}+Z_{2} \tan \phi_{1}\right)}{Z_{1}\left(Z_{2}-Z_{1} \tan \phi_{1} \tan \phi_{2}\right)-i Z_{0}\left(Z_{1} \tan \phi_{2}+Z_{2} \tan \phi_{1}\right)} .
$$

Since there is a reflective surface behind layer 2, the amplitude transmission coefficient $T=0$. Thus, the wave energy absorptance $A=1-|R|^{2}$, which means that in order to obtain a maximum value for $A$, we should minimize $|R|$. Such a task may be accomplished by choosing suitable materials for the two layers and their corresponding thicknesses. Here, to make our metasurface experimentally feasible, we use silicone rubber as layer 1 , whose mass density is $\rho_{1}=1300 \mathrm{~kg} / \mathrm{m}^{3}$ and sound velocity is $c_{1}=c_{\text {rubber }}(1-i \eta)$, where $c_{\text {rubber }}=337 \mathrm{~m} / \mathrm{s}$ and $\eta$ is the loss tangent factor, which is set to 0.11 , a typical value for polymer materials. ${ }^{47}$ Therefore, the impedance and the wavenumber in layer 1 are $Z_{1}=\frac{\rho_{1} c_{1}}{\rho_{0} c_{0}} Z_{0}=(0.2940-0.03234 i) Z_{0}$ and $k_{1}=\frac{c_{0}}{c_{1}} k_{0}=(4.3685+0.4805 i) k_{0}$, where $Z_{0}$ and $k_{0}$ are the impedance and the wavenumber in water, respectively.

Given the material of layer 1 , the remaining task is to determine the material parameters of layer 2 and the thickness of each layer to achieve nearly perfect absorption. For simplicity and without loss of generality, the thicknesses of layer 1 and layer 2 are chosen as $h_{1}=12 \mathrm{~m}$ and $h_{2}=6 \mathrm{~m}$, respectively. And, the working wavelength (best absorption performance) of the metasurface is set as $\lambda_{m}=118.4 \mathrm{~m}$ in water, which is roughly 6.6 times the total thickness of the metasurface. In this case, by solving Eq. (3), we find that layer 2 should possess a mass density $\rho_{2}=1.0332 \rho_{0}$ and a wave velocity $c_{2}=(0.5024-0.0458 i) c_{0}$. With such a combination of the material parameters and the thicknesses, we indeed get a nearly zero reflectance and nearly total absorptance $(A \approx 0.997)$ at $\lambda_{m}$.

\section{DESIGN AND SIMULATION}

However, the stringent requirement for the material parameters of layer 2 cannot be fulfilled by any existing natural materials. Thus, a clever design of an artificial structure with available materials which can produce effective material properties required by layer 2 is desirable: i.e., $\rho_{\text {eff }}=\rho_{2}$ and $c_{\text {eff }}=c_{2}$. We note that $\rho_{2}$ is close to $\rho_{0}$ and that $c_{2}$ is around half $c_{0}$, with a loss tangent around 0.09 . Such material properties may be achieved by using composite materials composed of common materials such as water and rubber. The simplest structure may be the grating structure shown in Fig. 1(b), where a layer of rubber cylinders is periodically distributed in water. An effective medium theory ${ }^{48}$ is adopted to determine the radius of the rubber cylinder $\left(r_{2}\right)$ and the spacing between two adjacent cylinders $\left(d_{2}\right)$. Then, an iterative method ${ }^{49}$ is further employed to optimize these two geometric parameters to guarantee nearly perfect absorption at $\lambda_{m}$.

The iterative method is developed from the parameter retrieval method. The desired results-i.e., the geometric parameters predicted by effective medium theory-are set as the starting point. With these parameters, we calculate the transmission and reflection coefficients of the rubber cylinder arrays and use the coefficients to retrieve a set of effective parameters. If the retrieved effective parameters are identical to the desired parameters, indicating that the predicted geometric parameters are accurate, the iteration stops. If the retrieved effective parameters are different from the desired ones (usually the difference is not large), we slightly adjust 
the geometric parameters and retrieve a new set of effective parameters. This step is repeated until the retrieved parameters are identical to the desired ones. Using this method, we obtain the optimized radius and spacing as $r_{2}=0.249 h_{2}=1.494 \mathrm{~m}$ and $d_{2}=h_{2}=6 \mathrm{~m}$.

Figure 2 shows the calculated wave energy reflectance $|R|^{2}$ and absorptance $\mathrm{A}=1-|R|^{2}$ of the designed metasurface for a normally incident plane wave. It clearly demonstrates that the absorptance reaches its maximum value ( $\sim 0.997$ ) around the working wavelength of $118.4 \mathrm{~m}$ (at the frequency $12.58 \mathrm{~Hz}$ ), exactly the wavelength $\lambda_{m}$ given in the design. At this absorption peak, the thickness of the metasurface accounts for only 0.15 of the wavelength, indicating that it is indeed subwavelength. Although the metasurface is designed for a particular wavelength, high absorption is manifested over a relatively broad frequency range in Fig. 2, which shows over $80 \%$ of absorption over a wavelength range of [105.82-130.86] $\mathrm{m}$ (corresponding to a frequency range of [11.386-14.080] Hz and a $21 \%$ relative bandwidth). This performance is indeed impressive for a metasurface with such a simple structure. The reason for the broadband absorption by this metasurface comes from its simple structure that does not rely on strong resonances. Unlike other popular resonating structures, our metasurface is designed from effective medium theory. The effective medium parameters of layer 2 do not change dramatically within the frequency range we are interested in, as shown in Fig. 3, and high absorption is achieved. Such a design principle is conceptually distinct from resonance-based metasurfaces, like Fabry-Perot resonance or Helmholtz resonance, which usually exhibit narrow bandwidth absorption.

To better demonstrate the physical mechanism of our metasurface, we plot in Fig. 4 the acoustic wave field distributions at the absorption peak wavelength $\lambda_{m}$. From Fig. 4(a), we can observe an antinode plane for the pressure wave at the reflective surface (i.e., the top surface), where the pressure takes the local maximum value indicated by the dark red color. Whereas almost in the middle of layer 1 (the rubber layer), the pressure takes the local minimum value denoted by the dark blue color. In both layers (layer 1 and

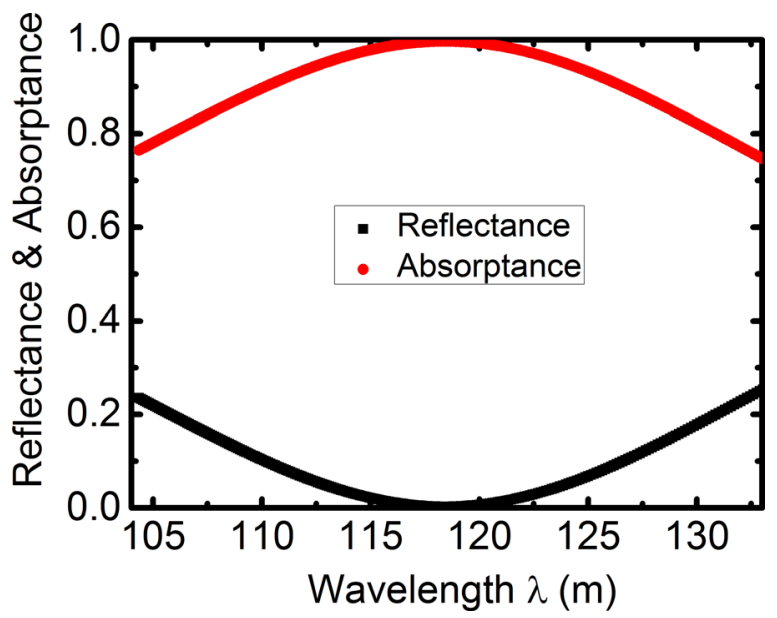

FIG. 2. Reflectance and absorptance of the designed metasurface as functions of the wavelength for the normally incident wave.

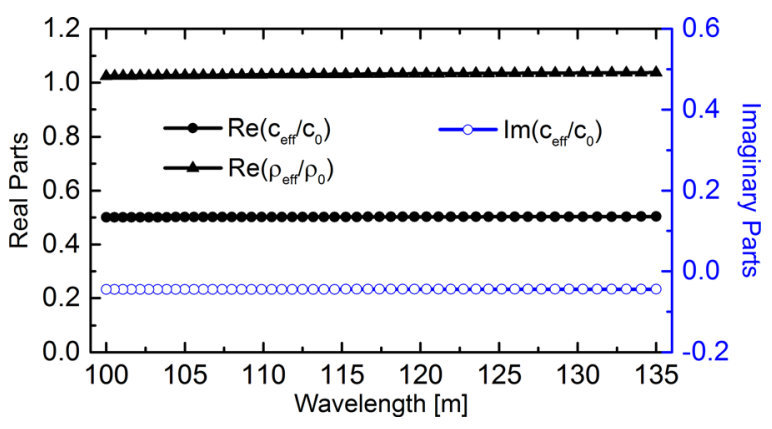

FIG. 3. Effective mass densities and wave velocities of the designed metasurface as functions of wavelength.

layer 2), the total pressure field-i.e., the sum of the incident and reflected waves-preserves the plane wave front, which in turn justifies the effective medium description of layer 2 , because the wave does not resolve the circular structure of the rubber cylinders. The time-averaged Poynting vector along the vertical direction is plotted in Fig. 4(b), which offers a clear picture of how the energy is dissipated within the metasurface. It unambiguously shows that the intensity of the acoustic wave is significantly attenuated after the wave passes through layer 1 and decays to nearly zero when the wave touches the rubber cylinder in layer 2. It is interesting to note that although the silicone rubber has a very small loss tangent $(\eta=0.11)$, the metasurface as a whole can work as an efficient absorber for low-frequency sound because of the destructive interference between the multiple reflections induced at the interfaces and the accurate prediction of effective medium theory in conjunction with the optimization method.

We also studied the absorption performance of the metasurface under oblique incidence. In Fig. 5(a), we plot the

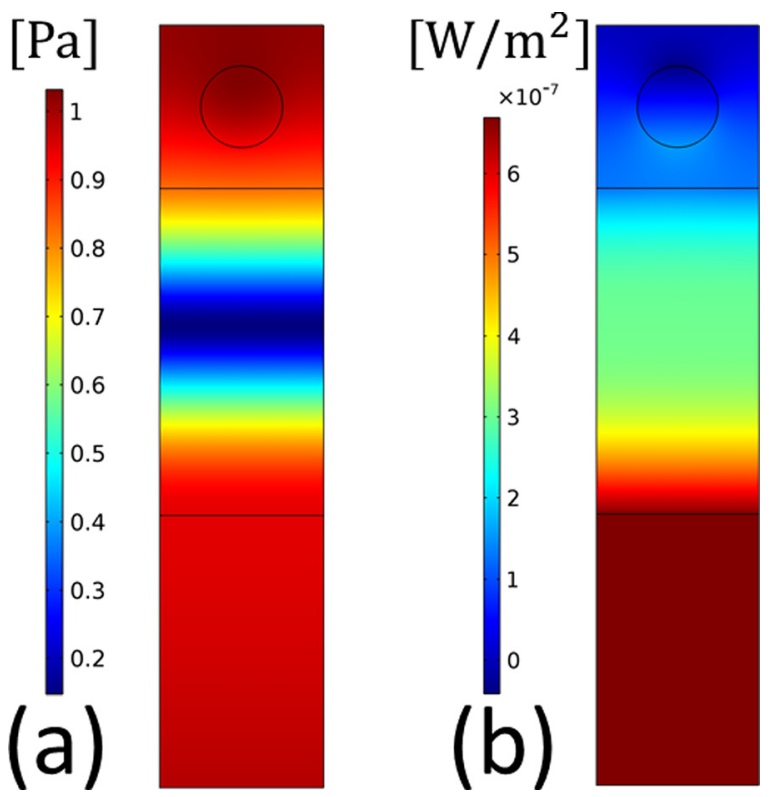

FIG. 4. Acoustic wave field distributions at the absorption peak frequency. (a) Absolute value of the pressure field. (b) Time-averaged Poynting vector along the y-direction. Red and blue denote maximum and minimum, respectively. The pressure amplitude of the incident wave is set as $1[\mathrm{~Pa}]$. 


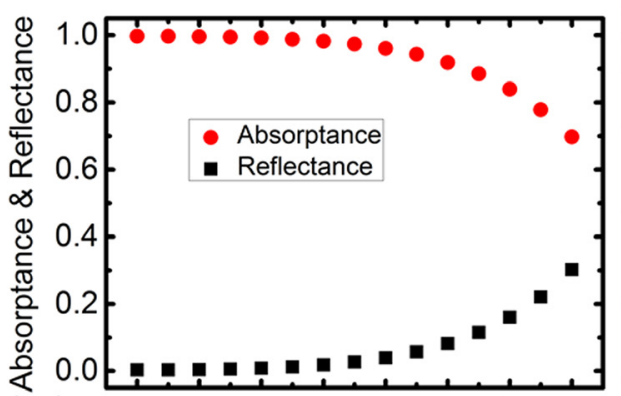

(a)

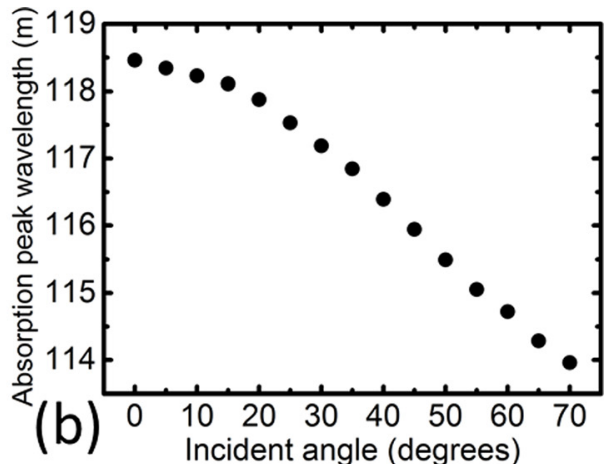

FIG. 5. Absorption performance of the metasurface under oblique incidence. (a) Maximum absorptance and minimum reflectance as functions of the incident angle. (b) Absorption peak wavelength as a function of the incident angle. maximum absorptance (together with minimum reflectance) of the acoustic wave energy at different incident angles. We find that the metasurface can absorb over $90 \%$ of the incident wave energy up to an incident angle of $50^{\circ}$. It should be noted that when the incident angle increases, the absorption peak wavelength shifts slightly, as indicated by Fig. 5(b). These results can be qualitatively understood from the following model for absorption as a function of incident angle.

When a plane wave is obliquely incident onto the metasurface, the amplitude reflection coefficient $R$ can be expressed as

$$
R=\frac{Z_{1}{ }^{\prime}\left(Z_{2}^{\prime}-Z_{1}{ }^{\prime} \tan \phi_{1} \tan \phi_{2}\right)+i Z_{0}{ }^{\prime}\left(Z_{1}{ }^{\prime} \tan \phi_{2}+Z_{2}{ }^{\prime} \tan \phi_{1}\right)}{Z_{1}{ }^{\prime}\left(Z_{2}{ }^{\prime}-Z_{1}{ }^{\prime} \tan \phi_{1} \tan \phi_{2}\right)-i Z_{0}{ }^{\prime}\left(Z_{1}{ }^{\prime} \tan \phi_{2}+Z_{2}{ }^{\prime} \tan \phi_{1}\right)}
$$

with the impedances given by $Z_{i}^{\prime}=Z_{i} / \cos \theta_{i}$, where $Z_{i}=\rho_{i} c_{i}$ $(i=1,2)$ is the specific acoustic impedance of each layer and $\theta_{i}$ is the angle between the wave vector in the $i$-th layer and the normal direction to the boundary of the layer. According to Snell's law, we have $k_{0} \sin \theta_{0}=k_{1} \sin \theta_{1}=k_{2} \sin \theta_{2}=k_{\|}$. Thus, $Z_{i}^{\prime}=Z_{i} / \sqrt{1-\left(\sin \theta_{i}\right)^{2}}$ and

$$
R=\frac{Z_{1}\left(Z_{2}-\sqrt{\frac{1-\left(\sin \theta_{2}\right)^{2}}{1-\left(\sin \theta_{1}\right)^{2}}} Z_{1} \tan \phi_{1} \tan \phi_{2}\right)+i Z_{0}\left(\sqrt{\frac{1-\left(\sin \theta_{2}\right)^{2}}{1-\left(\sin \theta_{0}\right)^{2}}} Z_{1} \tan \phi_{2}+\sqrt{\frac{1-\left(\sin \theta_{1}\right)^{2}}{1-\left(\sin \theta_{0}\right)^{2}}} Z_{2} \tan \phi_{1}\right)}{Z_{1}\left(Z_{2}-\sqrt{\frac{1-\left(\sin \theta_{2}\right)^{2}}{1-\left(\sin \theta_{1}\right)^{2}}} Z_{1} \tan \phi_{1} \tan \phi_{2}\right)-i Z_{0}\left(\sqrt{\frac{1-\left(\sin \theta_{2}\right)^{2}}{1-\left(\sin \theta_{0}\right)^{2}}} Z_{1} \tan \phi_{2}+\sqrt{\frac{1-\left(\sin \theta_{1}\right)^{2}}{1-\left(\sin \theta_{0}\right)^{2}}} Z_{2} \tan \phi_{1}\right)} .
$$

Obviously, the amplitude reflectance coefficient $R$ has a much more complicated expression compared with that for normal incidence, due to the appearance of terms in the form of $\sqrt{\frac{1-\left(\sin \theta_{i}\right)^{2}}{1-\left(\sin \theta_{j}\right)^{2}}}$. Thus, the original perfect absorption condition (which is designed for the normal incidence case) no longer brings about perfect absorption when applied to the oblique incidence cases, and a lower absorptance is expected. This is the reason for the gradually decreasing absorptance behavior when the incident angle increases from $0^{\circ}$.

\section{DISCUSSION}

In this work, we propose a new method for designing a highly absorptive metasurface for acoustic waves in water and present an $18 \mathrm{~m}$-thick metasurface as an example. We should note that the thickness can be further shrunk if the absorbing materials and/or working frequency are different. The loss tangent factor $\eta$ of the chosen absorbing material can affect the thickness of the metasurface. In the example we presented, silicone rubber $(\eta=0.11)$ was chosen as the absorbing material. We can of course choose other absorbing materials (with different $\eta$ ) to realize perfect absorption. In fact, even for plastics (such as rubber), their absorption properties vary considerably with molecular weight, additives and temperature. In principle, the larger the loss factor $\eta$, the smaller the thickness of the metasurface for a given working frequency, because the wave energy can be attenuated more intensively in an absorbing material with larger $\eta$. On the other hand, the working wavelength also determines the thickness of the metasurface. Since our design is scalable in principle, it is reasonable to expect a thinner metasurface for higher frequency absorption. In addition, most solids are dispersive and bear a larger loss tangent factor $\eta$ at higher frequencies than that at lower frequencies, implying that the thickness of the metasurface may be further reduced. But, one still needs to take impedance into account. Thus, for application in high frequencies, such as the ultrasound frequency regime, we may need to consider options involving different absorbing materials (either another kind of rubber, or a plastic material) that bear appropriate loss tangent factors and give matched impedance as well.

The advantages of the proposed design are the subwavelength thickness and non-resonant behavior compared to the existing methods adopted for underwater sound absorption by the engineering community, which usually involve utilizing locally resonant modes (such as air bubbles), multiplelayer viscoelastic materials, or wedge-based designs. For 
resonance-based absorbers, it is difficult to maintain a perfect absorption performance over a relatively broad bandwidth, due to the inherent characteristics of resonance mechanism. For multiple-layer viscoelastic materials or wedge-based structures, the designs are relatively complex, and an ultrathin solution is not easy to find.

\section{CONCLUSIONS}

To conclude, we propose a new design paradigm for an acoustic metasurface to efficiently absorb low-frequency waterborne sound. The metasurface is composed of two thin layers and is constructed from two common materials: water and rubber. We use an effective medium theory to predict the material and geometrical parameters of the thin layers and utilize an iterative method to further optimize these parameters in the design of the metasurface. Although the metasurface has a thickness of only 0.15 of the wavelength, nearly total absorptance $(99.7 \%)$ is obtained for normally incident sound waves, and very high absorptance is maintained for a relatively broad bandwidth, which is distinct from metasurfaces that are based on the resonance mechanism. Furthermore, the metasurface works very efficiently for oblique incident waves up to incident angles of $50^{\circ}$.

\section{ACKNOWLEDGMENTS}

This work was supported by the National Natural Science Foundation of China (Grant Nos. 11274120 and 11574087), the King Abdullah University of Science and Technology (KAUST) Baseline Research Fund, and the KAUST Office of Sponsored Research (OSR) under Award No. OSR-2016-CRG5-2950.

${ }^{1}$ N. Yu, P. Genevet, M. A. Kats, F. Aieta, J.-P. Tetienne, F. Capasso, and Z. Gaburro, Science 334, 333 (2011).

${ }^{2}$ X. Ni, N. K. Emani, A. V. Kildishev, A. Boltasseva, and V. M. Shalaev, Science 335, 427 (2012).

${ }^{3}$ A. V. Kildishev, A. Boltasseva, and V. M. Shalaev, Science 339, 1232009 (2013).

${ }^{4}$ Z. Liang and J. Li, Phys. Rev. Lett. 108, 114301 (2012).

${ }^{5}$ Y. Li, B. Liang, X.-Y. Zou, and J.-C. Cheng, Appl. Phys. Lett. 103, 063509 (2013).

${ }^{6}$ J. Zhao, B. Li, Z. N. Chen, and C.-W. Qiu, Appl. Phys. Lett. 103, 151604 (2013).

${ }^{7}$ Y. Xie, A. Konneker, B.-I. Popa, and S. A. Cummer, Appl. Phys. Lett. 103, 201906 (2013).

${ }^{8}$ J. Zhao, B. Li, Z. Chen, and C. W. Qiu, Sci. Rep. 3, 2537 (2013).

${ }^{9}$ Y. Li, B. Liang, Z. M. Gu, X. Y. Zou, and J. C. Cheng, Sci. Rep. 3, 2546 (2013).

${ }^{10}$ Y. Xie, W. Wang, H. Chen, A. Konneker, B.-I. Popa, and S. A. Cummer, Nat. Commun. 5, 5553 (2014).

${ }^{11}$ J. Mei and Y. Wu, New J. Phys. 16, 123007 (2014).

${ }^{12}$ Y. Li, X. Jiang, R.-Q. Li, B. Liang, X.-Y. Zou, L.-L. Yin, and J.-C. Cheng, Phys. Rev. Appl. 2, 064002 (2014).
${ }^{13}$ X. Jiang, B. Liang, R.-Q. Li, X.-Y. Zou, L.-L. Yin, and J.-C. Cheng, Appl. Phys. Lett. 105, 243505 (2014).

${ }^{14}$ W. Wang, Y. Xie, A. Konneker, B.-I. Popa, and S. A. Cummer, Appl. Phys. Lett. 105, 101904 (2014).

${ }^{15}$ K. Tang, C. Qiu, M. Ke, J. Lu, Y. Ye, and Z. Liu, Sci. Rep. 4, 6517 (2014).

${ }^{16}$ Y. Cheng, C. Zhou, B. Yuan, D. Wu, Q. Wei, and X. Liu, Nat. Mater. 14, 1013 (2015).

${ }^{17}$ D. L. Sounas, R. Fleury, and A. Alù, Phys. Rev. Appl. 4, 014005 (2015).

${ }^{18}$ Y. Li, X. Jiang, B. Liang, J.-C. Cheng, and L. Zhang, Phys. Rev. Appl. 4, 024003 (2015).

${ }^{19}$ K. Tang, C. Qiu, J. Lu, M. Ke, and Z. Liu, J. Appl. Phys. 117, 024503 (2015).

${ }^{20}$ Y. Li, S. Qi, and M. B. Assouar, New J. Phys. 18, 043024 (2016).

${ }^{21}$ R. A. Jadhali and Y. Wu, Appl. Phys. Lett. 108, 031902 (2016).

${ }^{22}$ C. Shen, Y. Xie, J. Li, S. A. Cummer, and Y. Jing, Appl. Phys. Lett. 108, 223502 (2016).

${ }^{23}$ W. Wang, Y. Xie, B.-I. Popa, and S. A. Cummer, J. Appl. Phys. 120, 195103 (2016).

${ }^{24}$ S. Qi, Y. Li, and B. Assouar, Phys. Rev. Appl. 7, 054006 (2017).

${ }^{25}$ X. Bai, C. Qiu, X. Wen, S. Peng, M. Ke, and Z. Liu, AIP Adv. 7, 055001 (2017).

${ }^{26}$ Y. Bai, H. Jia, W. Lu, P. Ji, and J. Yang, Sci. Rep. 7, 705 (2017).

${ }^{27}$ G. Ma and P. Sheng, Sci. Adv. 2, e1501595 (2016).

${ }^{28}$ S. A. Cummer, J. Christensen, and A. Alu, Nat. Rev. Mater. 1, 16001 (2016).

${ }^{29}$ M. Yang and P. Sheng, Annu. Rev. Mater. Res. 47, 83 (2017).

${ }^{30}$ J. Mei, G. Ma, M. Yang, Z. Yang, W. Wen, and P. Sheng, Nat. Commun. 3, 756 (2012).

${ }^{31}$ G. Ma, M. Yang, S. Xiao, Z. Yang, and P. Sheng, Nat. Mater. 13, 873 (2014).

${ }^{32}$ J. Z. Song, P. Bai, Z. H. Hang, and Y. Lai, New J. Phys. 16, 033026 (2014).

${ }^{33}$ J. R. Piper, V. Liu, and S. Fan, Appl. Phys. Lett. 104, 251110 (2014).

${ }^{34}$ X. Cai, Q. Guo, G. Hu, and J. Yang, Appl. Phys. Lett. 105, 121901 (2014).

${ }^{35}$ M. Yang, Y. Li, C. Meng, C. Fu, J. Mei, Z. Yang, and P. Sheng, C.R. Mec. 343, 635 (2015).

${ }^{36}$ M. Yang, C. Meng, C. Fu, Y. Li, Z. Yang, and P. Sheng, Appl. Phys. Lett. 107, 104104 (2015).

${ }^{37}$ A. Merkel, G. Theocharis, O. Richoux, V. Romero-García, and V. Pagneux, Appl. Phys. Lett. 107, 244102 (2015).

${ }^{38}$ Y. Duan, J. Luo, G. Wang, Z. H. Hang, B. Hou, J. Li, P. Sheng, and Y. Lai, Sci. Rep. 5, 12139 (2015).

${ }^{39}$ Y. Li and B. M. Assouar, Appl. Phys. Lett. 108, 063502 (2016).

${ }^{40}$ S. Qi, M. Oudich, Y. Li, and B. Assouar, Appl. Phys. Lett. 108, 263501 (2016).

${ }^{41}$ J. Li, W. Wang, Y. Xie, B.-I. Popa, and S. A. Cummer, Appl. Phys. Lett. 109, 091908 (2016).

${ }^{42}$ V. Romero-García, G. Theocharis, O. Richoux, A. Merkel, V. Tournat, and V. Pagneux, Sci. Rep. 6, 19519 (2016).

${ }^{43}$ V. Romero-García, G. Theocharis, O. Richoux, and V. Pagneux, J. Acoust. Soc. Am. 139, 3395 (2016).

${ }^{44}$ Y. Li, C. Shen, Y. Xie, J. Li, W. Wang, S. A. Cummer, and Y. Jing, Phys. Rev. Lett. 119, 035501 (2017).

${ }^{45}$ M. Yang, S. Chen, C. Fu, and P. Sheng, Mater. Horiz. 4, 673 (2017).

${ }^{46}$ V. Leroy, A. Strybulevych, M. Lanoy, F. Lemoult, A. Tourin, and J. H. Page, Phys. Rev. B 91, 020301 (2015).

${ }^{47} \mathrm{Y}$. Zhang, "Measuring acoustic attenuation of polymer materials using drop ball test," Master thesis (2013).

${ }^{48} \mathrm{X}$. Zhang, R. McPhedran, and Y. Wu, "Effective medium theory for grating structures" (unpublished).

${ }^{49}$ X. Zhang and Y. Wu, Opt. Express 25, 4860 (2017). 